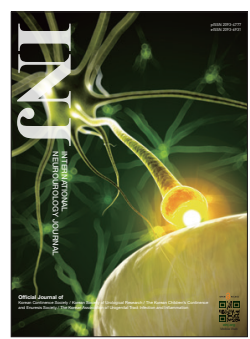

\title{
Effects of Pelvic Floor Muscle Exercise on Urinary Incontinence in Elderly Women With Cognitive Impairment
}

\author{
Bo Ae Lee ${ }^{1, *}$, Su Jin Kim ${ }^{2, *}$, Don Kyoung Choi ${ }^{3}$, Ohseong Kwon ${ }^{3}$, Hae Ri Na ${ }^{4}$, Sung Tae Cho \\ ${ }^{1}$ Department of Rehabilitation Medicine, Pusan National University School of Medicine, Busan, Korea \\ ${ }^{2}$ Department of Urology, Seoul St. Mary's Hospital, College of Medicine, The Catholic University of Korea, Seoul, Korea \\ ${ }^{3}$ Department of Urology, Hallym University Kangnam Sacred Heart Hospital, Hallym University College of Medicine, Seoul, Korea \\ ${ }^{4}$ Department of Neurology, Bobath Memorial Hospital, Seongnam, Korea
}

Purpose: Pelvic floor muscle exercise (PFME) is a therapeutic option for urinary incontinence (UI). However, studies of the efficacy of PFME on UI in patients with cognitive impairment (CI) are lacking. Therefore, we evaluated the effect of PFME on UI in elderly women with mild CI.

Methods: A total of 150 women with mild CI or Alzheimer disease and UI were screened using the International Consultation on Incontinence Questionnaire-Short Form (ICIQ-SF). Cognitive function and behavioral symptoms were evaluated by the Mini-Mental State Examination and Barthel's Activities of Daily Living. The patients were randomly divided into a control group $(n=46)$ and a PFME group ( $n=52,6$ sessions of PFME for 12 weeks). The primary outcome was the change in UI episodes measured with a frequency volume chart (FVC). The secondary outcomes were other FVC parameters and the ICIQ-SF scores.

Results: A total of 82 women (control group: 40 and PFME group: 42) completed the study. After 12 weeks of PFME, the mean number of UI episodes per 24 hours decreased by 1.6 (from 3.3 to 1.7) in the PFME group and by 0.5 (from 3.4 to 2.9 ) in the control group $(\mathrm{P}<0.001$ between groups). The mean number of micturition episodes and total ICIQ-SF scores improved in the PFME group to a significantly greater extent than in the control group $(\mathrm{P}<0.001)$.

Conclusions: Supervised PFME can be a good therapeutic option for improving UI in elderly women with CI.

Keywords: Urinary Incontinence; Pelvic Floor; Cognitive Dysfunction; Aged

- Research Ethics: This study was approved by Institutional Review Board of the Hallym University Kangnam Sacred Heart Hospital (2011-10-89). Written informed consent was obtained from all patients or legal guardians in accordance with the Declaration of Helsinki.

- Conflict of Interest: SJK, a member of the Editorial Board of INJ, is the co-first author of this article. However, she played no role whatsoever in the editorial evaluation of this article or the decision to publish it. No potential conflict of interest relevant to this article was reported.

\section{INTRODUCTION}

Cognitive impairment (CI) and urinary incontinence (UI) are common health problems in the elderly. Both CI and UI are important factors that limit normal activities in elderly women and decrease their day-to-day quality of life [1-3]. Moreover, the prevalence of UI has been found to be higher in the elderly with dementia than in those without dementia, and it is well known that cognitive dysfunction is a major risk factor for UI $[4,5]$. Erekson et al. [6] noted that CI was common in women over 65 years and older with pelvic floor dysfunction, such as UI and pelvic organ prolapse.
Corresponding author: Sung Tae Cho (iD https://orcid.org/0000-0002-4691-6159 Department of Urology, Hallym University Kangnam Sacred Heart Hospital, Hallym University College of Medicine, 1 Singil-ro, Yeongdeungpo-gu, Seoul 07441, Korea E-mail: cst326@paran.com / Tel: +82-2-829-5198 / Fax: +82-2-846-5198 *Bo Ae Lee and Su Jin Kim contributed equally to this study as co-first authors. Submitted: August 10, 2017 / Accepted after revision: November 20, 2017
(5) This is an Open Access article distributed under the terms of the Creative Commons Attribution Non-Commercial License (http://creativecommons.org/licenses/by-nc/4.0/) which permits unrestricted non-commercial use, distribution, and reproduction in any medium, provided the original work is properly cited. 
UI in elderly women with CI or dementia is treated differently from how it is treated in women with normal cognitive function, because women with CI need assistance from their caregivers to carry out many activities of daily life, such as using the toilet. However, there are no standard and effective treatment guidelines for elderly individuals with CI and UI $[7,8]$. In addition, the polypharmacy that is frequently observed in the elderly with CI or dementia is an important factor in deciding on treatment with anticholinergics to reduce UI [9]. Several reports have investigated the adverse effects of antimuscarinics on cognitive function, although cognitive function usually recovers after the cessation of antimuscarinic treatment. Moreover, the impairment of cognitive function associated with antimuscarinics is greater in elderly dementia patients who concomitantly take cholinesterase inhibitors [10]. Therefore, treatment with antimuscarinics should be prescribed based on each patient's clinical circumstances, and treatment methods other than antimuscarinics should be considered to minimize cognitive dysfunction in elderly women.

Pelvic floor muscle exercise (PFME) is a nonpharmacological and nonsurgical treatment to reduce UI by strengthening the pelvic floor muscle, because UI is associated with weakened pelvic floor support due to aging [11]. Previously, several studies showed that the effects of PFME were similar in premenopausal and postmenopausal women with UI and that PFME could reduce UI in elderly women $[12,13]$. Thus, PFME is an option for the elderly with UI who are at risk of CI due to polypharmacy. However, little research has been conducted into the effects of PFME on UI in elderly women with CI, and therefore it is necessary to investigate the impact of PFME on UI in elderly women with CI. PFME cannot be applied to elderly women with severely impaired cognitive function because the outcome of PFME depends on the patient's compliance. Thus, in the present study, the effect of PFME on the improvement of UI was evaluated in elderly women with mild CI.

\section{MATERIALS AND METHODS}

\section{Study Design}

Female patients with mild CI or mild Alzheimer disease (AD) and UI were screened. Ninety-eight of the 150 patients who agreed to participate in this study were enrolled. This study was approved by Institutional Review Board of the Hallym University Kangnam Sacred Heart Hospital (2011-10-89). Written informed consent was obtained from all patients or legal guardians in accordance with the Declaration of Helsinki.

Mild CI and $\mathrm{AD}$ were diagnosed by a neurologist and neuropsychologist based on the National Institute of Neurological Disorders and Stroke-Alzheimer Disease and Related Disorders criteria. Cognitive function and behavioral symptoms were evaluated by the Mini-Mental State Examination and Barthel's Activities of Daily Living. UI was evaluated with a frequency volume chart (FVC), including a urinary sensation scale to evaluate the degree of urgency for 3 days, and the International Consultation on Incontinence Questionnaire-Short Form (ICIQ-SF). Exclusion criteria included severe dementia with

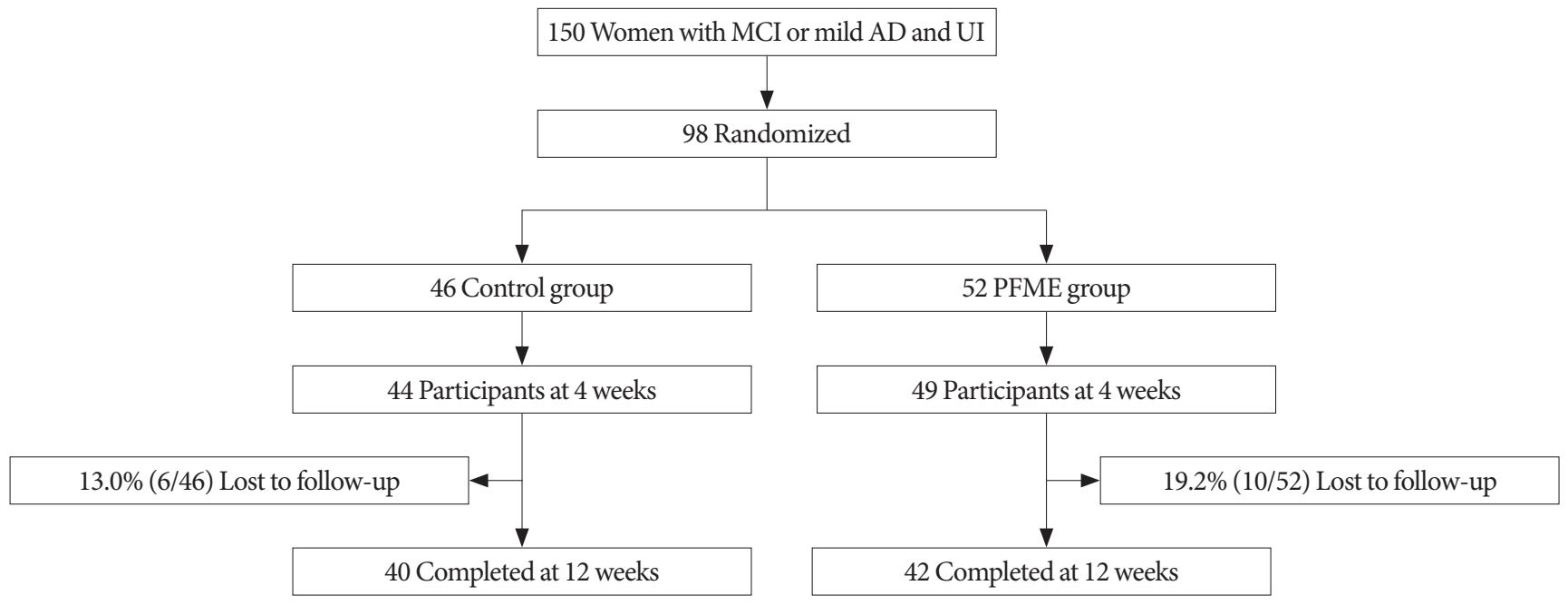

Fig. 1. Study flowchart. MCI, mild cognitive impairment; AD, Alzheimer disease; UI, urinary incontinence; PFME, pelvic floor muscle exercise. 
behavioral disturbances, previous pelvic surgery, urinary tract infections, pelvic organ prolapse, or an inability to communicate and contract the pelvic floor muscle. Patients previously or currently treated with anticholinergic medications or beta-3 agonists were also excluded.

The patients were randomly divided into 2 groups. The PFME group $(n=52)$ received 6 sessions of PFME (60 minutes per session at 2-week intervals) with vaginal palpation and verbal feedback over the course of 12 weeks. PFME instructions were provided by an expert physiotherapist, and the patients received education about the mechanism of UI, the anatomy of the pelvic floor muscle, and bladder training (BT) with urge suppression. Between each session, all participants performed PFME at home. The control group $(n=46)$ received education about the mechanism of UI, the anatomy of the pelvic floor muscle, and conservative treatment such as lifestyle modification and BT. Follow-up with an FVC and the ICIQ-SF was scheduled at 4 and 12 weeks after the start of PFME. The primary outcome was the change in UI episodes analyzed with FVC. The secondary outcomes were other parameters analyzed using the FVC (degree of urgency, number of micturition episodes, and voided volume) and ICIQ-SF scores (Fig. 1).

\section{Statistical Analysis}

The paired-samples $t$-test was used to compare the difference in UI episodes within each group. The mean change in UI frequency (baseline minus follow-up) was compared between groups using the independent-samples t-test. Statistical analysis was carried out using SPSS ver. 17.0 (SPSS Inc., Chicago, IL, USA) and P-values $<0.05$ were considered to indicate statistical significance.

\section{RESULTS}

The mean age of the 98 women included in this study was $75.1 \pm 4.1$ years. Of the 98 women, $10(19.2 \%)$ in the PFME group and $6(13.0 \%)$ in the control group dropped out. A total of 82 women completed the entire 12 weeks of the study. Patients' characteristics and ICIQ-SF questionnaire results were not significantly different between the 2 groups at baseline (Tables 1,2).

At 4 weeks, both the PFME and control groups showed no significant differences in the mean number of UI episodes, micturition episodes, and voided volume compared with baseline. The maximum urgency intensity was not significantly different compared with baseline. In addition, the total ICIQ-SF scores of both groups were not significantly different. Table 3 and Fig. 2 show changes in the parameters evaluated with FVC after 12 weeks of treatment. UI episodes in the PFME group significantly decreased in comparison to baseline $(\mathrm{P}<0.01)$. The control group also showed a significant decrease in UI episodes compared to baseline $(\mathrm{P}<0.05)$. Specifically, the mean number of UI episodes in the PFME group significantly decreased by 1.6 (from 3.3 at baseline to 1.7 at 12 weeks), and this decrease was significantly greater than the decrease of $0.5 \mathrm{ob}-$ served in the control group (from 3.4 at baseline to 2.9 at 12 weeks) $(\mathrm{P}<0.001$ between groups). In addition, the mean number of micturition episodes significantly decreased only in the PFME group at 12 weeks compared with baseline $(\mathrm{P}<0.01)$. Specifically, the mean number of micturition episodes in the PFME group decreased by 2.5 (from 8.1 at baseline to 5.6 at 12 weeks), and this decrease was significantly greater than the decrease of 0.1 observed in the control group (from 8.7 at baseline to 8.6 at 12 weeks) $(\mathrm{P}<0.001$ between groups). The voided vol-

Table 1. Baseline characteristics

\begin{tabular}{lccc}
\hline Characteristic & Control $(\mathrm{n}=40)$ & PFME $(\mathrm{n}=42)$ & P-value \\
\hline Age $(\mathrm{yr})$ & $75.6 \pm 4.0$ & $74.5 \pm 4.1$ & 0.22 \\
Parity (times) & $3.0 \pm 0.7$ & $2.9 \pm 0.7$ & 0.78 \\
Body mass index $\left(\mathrm{kg} / \mathrm{m}^{2}\right)$ & $22.0 \pm 1.8$ & $22.5 \pm 2.1$ & 0.21 \\
Symptom duration $(\mathrm{yr})$ & $4.3 \pm 1.5$ & $4.9 \pm 1.7$ & 0.09 \\
Mild CI/mild AD & $28 / 12$ & $24 / 18$ & 0.26 \\
MMSE & $23.0 \pm 2.8$ & $23.4 \pm 2.5$ & 0.46 \\
ADL & $19.2 \pm 0.8$ & $19.2 \pm 1.7$ & 0.84
\end{tabular}

Values are presented as mean \pm standard deviation or number.

PFME, pelvic floor muscle exercise; CI, cognitive impairment; AD, Alzheimer disease; MMSE, Mini-Mental State Examination; ADL, Barthel's Activities of Daily Living. 
Table 2. ICIQ-SF questionnaire score at baseline

\begin{tabular}{lcc}
\hline ICIQ-SF questionnaire & Control $(\mathrm{n}=40)$ & PFME $(\mathrm{n}=42)$ \\
\hline How often do you leak urine? & & $0(0)$ \\
Never & $0(0)$ & $2(4.7)$ \\
About once a week or less often & $7(17.5)$ & $14(33.3)$ \\
Two or three times a week & $14(35.0)$ & $18(42.9)$ \\
About once a day & $10(25.0)$ & $7(16.7)$ \\
Several times a day & $6(15.0)$ & $1(2.4)$ \\
All the time & $3(7.5)$ & 0.19 \\
How much urine do you usually leak? & & $11(26.2)$ \\
None & $0(0)$ & $20(47.6)$ \\
A small amount & $13(32.5)$ & $11(26.2)$ \\
A moderate amount & $23(57.5)$ & \\
A large amount & $4(10.0)$ & $6.1 \pm 1.6$ \\
How much dose leaking urine interfere with your everyday life? & & \\
Between 0 (not at all) and 10 (a great deal) & $6.0 \pm 0.3$ & $20(47.6)$ \\
Types of UI & & $12(28.6)$ \\
Stress UI & $15(37.5)$ & $7(16.7)$ \\
Urge UI & $13(32.5)$ & $1(2.4)$ \\
Mixed UI & $7(17.5)$ & $2(4.8)$ \\
Enuresis & $3(7.5)$ & 0.17 \\
Unexplained & $2(5.0)$ & 0.79 \\
\hline
\end{tabular}

Values are presented as number (\%) or mean \pm standard deviation.

ICIQ-SF, International Consultation on Incontinence Questionnaire-Short Form; PFME, pelvic floor muscle exercise; UI, urinary incontinence.

Table 3. Changes of UI episodes and other parameters analyzed with FVC at 12 weeks

\begin{tabular}{|c|c|c|c|c|c|}
\hline \multirow{2}{*}{ Parameter } & \multicolumn{2}{|c|}{ Control $(n=40)$} & \multicolumn{2}{|c|}{$\operatorname{PFME}(n=42)$} & \multirow{2}{*}{ P-value } \\
\hline & Baseline & 12 Weeks & Baseline & 12 Weeks & \\
\hline UI episodes (number per day) & $3.4 \pm 1.2$ & $2.9 \pm 1.1^{*}$ & $3.3 \pm 1.1$ & $1.7 \pm 1.0^{\star *}$ & \\
\hline Change from baseline to follow-up & \multicolumn{2}{|c|}{$-0.5 \pm 1.3$} & \multicolumn{2}{|c|}{$-1.6 \pm 1.3$} & $<0.001$ \\
\hline Micturition (number per day) & $8.7 \pm 1.9$ & $8.6 \pm 2.0$ & $8.1 \pm 2.4$ & $5.6 \pm 0.9^{* *}$ & \\
\hline Change from baseline to follow-up & \multicolumn{2}{|c|}{$-0.1 \pm 1.4$} & \multicolumn{2}{|c|}{$-2.5 \pm 2.8$} & $<0.001$ \\
\hline Maximum urgency intensity & $2.4 \pm 1.0$ & $2.2 \pm 1.1$ & $2.3 \pm 1.0$ & $2.2 \pm 1.1$ & \\
\hline Change from baseline to follow-up & \multicolumn{2}{|c|}{$-0.2 \pm 1.0$} & \multicolumn{2}{|c|}{$-0.1 \pm 0.9$} & 0.93 \\
\hline Voided volume (mL) & $190.3 \pm 129.0$ & $205.0 \pm 125.9$ & $163.5 \pm 93.6$ & $183.4 \pm 98.0^{*}$ & \\
\hline Change from baseline to follow-up & \multicolumn{2}{|c|}{$-14.7 \pm 45.3$} & \multicolumn{2}{|c|}{$-19.9 \pm 52.4$} & 0.63 \\
\hline
\end{tabular}

Values are presented as mean \pm standard deviation.

UI, urinary incontinence; FVC, frequency volume chart; PFME, pelvic floor muscle exercise.

${ }^{\star} \mathrm{P}<0.05$ compared with baseline within group. ${ }^{*} \mathrm{P}<0.01$ compared with baseline within group.

ume in the PFME group at 12 weeks was significantly greater than the voided volume before treatment $(\mathrm{P}<0.05)$. However, there was no significant difference in the change of voided volume between the PFME and control groups at 12 weeks. No significant difference was found in the maximum urgency intensity within each group at 12 weeks compared with baseline or between the 2 groups. The ICIQ-SF scores at 12 weeks $(9.2 \pm 5.9)$ significantly decreased in comparison to baseline
$(11.8 \pm 3.7)$ and 4 weeks $(11.3 \pm 3.9)$ in the PFME group $(\mathrm{P}<0.01)$ (Fig. 2). However, the control group did not exhibit a significant decrease in the total ICIQ-SF scores at 12 weeks. Moreover, the total ICIQ-SF scores in the PFME group significantly decreased in comparison to the control group $(\mathrm{P}<0.001)$ (Fig. 2). 


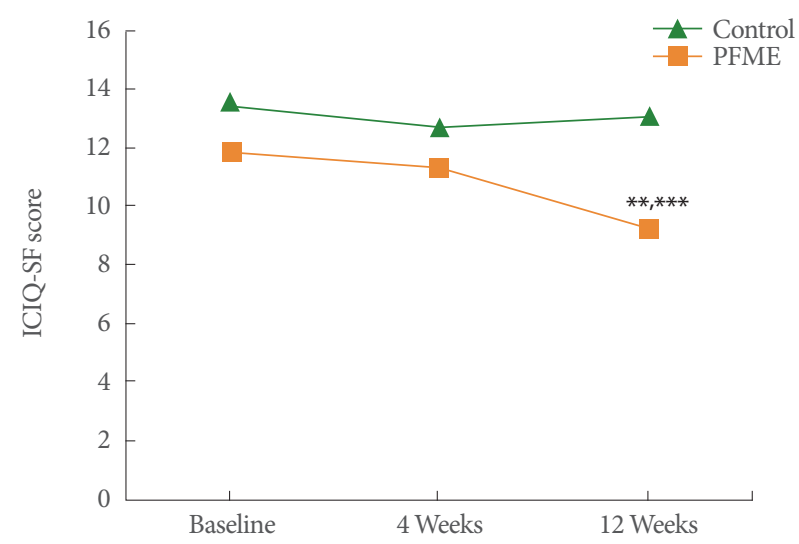

Fig. 2. Changes in the total ICIQ-SF scores. ICIQ-SF, International Consultation on Incontinence Questionnaire-Short Form; PFME, pelvic floor muscle exercise. ${ }^{* *} \mathrm{P}<0.01$ compared with baseline and 4 weeks within the group. ${ }^{* *} \mathrm{P}<0.001 \mathrm{com}-$ pared with the control group.

\section{DISCUSSION}

The present study demonstrated the efficacy of PFME for improving UI in elderly women with mild CI or AD. A significant reduction in the number of UI and micturition episodes was observed in the FVC and a significant improvement in the subjective symptoms evaluated by the ICIQ-SF was noted after 12 weeks of PFME. These results suggest that PFME delivered by a well-trained instructor could be an effective treatment option for reducing UI in elderly women with mild CI or dementia.

UI is a common disorder in the elderly with cognitive dysfunction or dementia. Moreover, the type and severity of dementia may affect the degree of UI $[14,15]$. In addition, a recent study showed that the prevalence of mild CI and dementia was $15 \%$ and $17 \%$, respectively, in women over 55 years with pelvic organ dysfunction, including UI [16]. These findings imply that a close relationship exists between UI and cognitive function and that CI influences the occurrence of UI. Therefore, the treatment of UI should take cognitive function into account, as well as the characteristics of UI. Nonpharmacological treatment, such as behavioral and physical therapy, is a treatment option for managing UI. However, little research has investigated the efficacy of nonpharmacological treatment of UI in patients with CI and dementia. Furthermore, most previous studies of conservative therapy evaluated the effect of scheduled or prompt voiding [17-19], whereas no studies have investigated PFME in patients with CI or dementia. Thus, the present study, in which PFME was used to treat UI, is the first prospective randomized trial of this treatment modality in elderly women with CI or dementia. Stress, urge, and mixed UI were observed in women with mild CI or mild AD according to the ICIQ-SF questionnaire, and improvement of UI was observed at 12 weeks after PFME, regardless of the type of UI. However, no significant improvement in UI was noted at 4 weeks, and we assumed that the absence of improvement in UI after 4 weeks of PFME may have been associated with the characteristics of the patients that influenced the severity of UI. A previous study reported that 6 weeks of PFME combined with BT led to a significant improvement in UI [20]. They observed improvements in UI after a short-term treatment, but we did not observe a significant decrease in the number of UI episodes at 4 weeks. This discrepancy may have been due to differences between the studies in terms of the characteristics of the subjects. The women who were treated with PFME and BT in the study by Kaya et al. [20] were younger (stress UI, $48.1 \pm 10.4$ years; urge UI, $46.3 \pm 11.6$ years; and mixed UI, $50.2 \pm 9.2$ years) than those treated with PFME in the present study (stress, urge, or mixed UI, $74.5 \pm 4.1$ years). In addition, the mean number of UI episodes before treatment, as measured by the FVC, was higher in the present study ( 3.3 per day) than in the women who participated in the study by Kaya et al. (1.0 per day) [20]. Thus, a longer duration of PFME was presumably necessary to achieve a reduction in the number of UI episodes because the women in the present study were older and suffered from severe UI. Sherburn et al. [21] reported similar results after 12 weeks of PFME for the improvement of stress UI in elderly women of a similar age (71.6 \pm 4.73 years) and with similar total ICIQ-SF scores $(10.4 \pm 5.0)$ to those of the women in present study (age, $74.5 \pm 4.1$ years and total ICIQ-SF score, 11.8 \pm 3.7 ). In comparison with baseline, stress UI significantly improved at 12 weeks after treatment. Similar changes in the total ICIQ-SF scores and micturition frequency were observed both in the study of Sherburn et al. [21] and in our study.

Although the elderly women in the present study had CI, the effects of treatment were similar to those observed in elderly women with normal cognitive function in a previous report [21]. We suggest that this can be explained in terms of how the patients performed PFME. Women with UI, both in the study by Sherburn et al. [21] and in our study, received PFME under the supervision of an expert physiotherapist, and the patients were able to continue PFME effectively. A review comparing therapeutic outcomes according to the type of PFME showed that regular supervision by an expert is important for improve- 
ments in UI and the patient's satisfaction [22]. Moreover, regular evaluation and feedback by the instructor helps to build a patient's sense of efficacy regarding PFME and to maintain adherence to the treatment [23]. Therefore, regular check-ups and assurances by the physiotherapist in this study contributed to the significant improvements in UI in elderly women with CI. Furthermore, the elderly women in this study had mild CI, meaning that they could be successfully instructed and could accurately follow the instructions of the physiotherapist.

This is the first study to present the therapeutic effects of PFME in elderly women with CI; however, this study has some limitations, and certain considerations should be taken into account for clinical practice. In this study, we only evaluated the efficacy of PFME in elderly women with mild CI. Although PFME cannot be applied to patients with severe CI who cannot be meaningfully instructed, it is necessary to evaluate the effects of PFME in patients with various degrees of CI selected using more detailed criteria. In addition, a tailored PFME protocol according to the status of cognitive function should be developed because no consensus exists regarding a standard protocol. We did not analyze the efficacy of PFME according to type of UI because PFME is well-known to be effective for improving stress, urge, and mixed UI. However, it is possible that PFME could have different effects depending on the type of UI. Thus, a further study with a subgroup analysis is necessary.

In this study, we observed that supervised PFME was effective for improving UI in elderly women with mild CI. Therefore, we suggest that PFME is a useful therapeutic option for elderly women with CI and UI, especially for patients who are at risk of $\mathrm{CI}$ associated with polypharmacy.

\section{ACKNOWLEDGEMENTS}

This work was presented by Sung Tae Cho (corresponding author) and received the Conservative Management Award at the 2016 meeting of the International Continence Society.

\section{REFERENCES}

1. Panza F, Solfrizzi V, Barulli MR, Santamato A, Seripa D, Pilotto A, et al. Cognitive frailty: a systematic review of epidemiological and neurobiological evidence of an age-related clinical condition. Rejuvenation Res 2015;18:389-412.

2. Markland AD, Vaughan CP, Johnson TM 2nd, Burgio KL, Goode PS. Incontinence. Med Clin North Am 2011;95:539-54, x-xi.
3. Jung HB, Kim HJ, Cho ST. A current perspective on geriatric lower urinary tract dysfunction. Korean J Urol 2015;56:266-75.

4. Campbell AJ, Reinken J, McCosh L. Incontinence in the elderly: prevalence and prognosis. Age Ageing 1985;14:65-70.

5. Offermans MP, Du Moulin MF, Hamers JP, Dassen T, Halfens RJ. Prevalence of urinary incontinence and associated risk factors in nursing home residents: a systematic review. Neurourol Urodyn 2009;28:288-94.

6. Erekson EA, Fried TR, Martin DK, Rutherford TJ, Strohbehn K, Bynum JP. Frailty, cognitive impairment, and functional disability in older women with female pelvic floor dysfunction. Int Urogynecol J 2015;26:823-30.

7. Parker D, Mills S, Abbey J. Effectiveness of interventions that assist caregivers to support people with dementia living in the community: a systematic review. Int J Evid Based Healthc 2008;6:137-72.

8. Drennan VM, Greenwood N, Cole L, Fader M, Grant R, Rait G, et al. Conservative interventions for incontinence in people with dementia or cognitive impairment, living at home: a systematic review. BMC Geriatr 2012;12:77.

9. Clague F, Mercer SW, McLean G, Reynish E, Guthrie B. Comorbidity and polypharmacy in people with dementia: insights from a large, population-based cross-sectional analysis of primary care data. Age Ageing 2017;46:33-9.

10. Orme S, Morris V, Gibson W, Wagg A. Managing urinary incontinence in patients with dementia: pharmacological treatment options and considerations. Drugs Aging 2015;32:559-67.

11. Parker WP, Griebling TL. Nonsurgical treatment of urinary incontinence in elderly women. Clin Geriatr Med 2015;31:471-85.

12. Betschart C, Mol SE, Lütolf-Keller B, Fink D, Perucchini D, Scheiner D. Pelvic floor muscle training for urinary incontinence: a comparison of outcomes in premenopausal versus postmenopausal women. Female Pelvic Med Reconstr Surg 2013;19:219-24.

13. Nygaard CC, Betschart C, Hafez AA, Lewis E, Chasiotis I, Doumouchtsis SK. Impact of menopausal status on the outcome of pelvic floor physiotherapy in women with urinary incontinence. Int Urogynecol J 2013;24:2071-6.

14. Ransmayr GN, Holliger S, Schletterer K, Heidler H, Deibl M, Poewe $\mathrm{W}$, et al. Lower urinary tract symptoms in dementia with Lewy bodies, Parkinson disease, and Alzheimer disease. Neurology 2008;70:299-303.

15. Lee SH, Cho ST, Na HR, Ko SB, Park MH. Urinary incontinence in patients with Alzheimer's disease: relationship between symptom status and urodynamic diagnoses. Int J Urol 2014;21:683-7.

16. Kunkle CM, Abernethy MG, Van Tongeren LR, Fashokun TB, Wright EJ, Chen CC. Prevalence of cognitive impairment in older 
women with pelvic floor disorders. Int Urogynecol J 2017;28:164550 .

17. Adkins VK, Mathews RM. Prompted voiding to reduce incontinence in community-dwelling older adults. J Appl Behav Anal 1997;30:153-6.

18. Jirovec MM, Templin T. Predicting success using individualized scheduled toileting for memory-impaired elders at home. Res Nurs Health 2001;24:1-8.

19. Engberg S, Sereika SM, McDowell BJ, Weber E, Brodak I. Effectiveness of prompted voiding in treating urinary incontinence in cognitively impaired homebound older adults. J Wound Ostomy Continence Nurs 2002;29:252-65.

20. Kaya S, Akbayrak T, Gursen C, Beksac S. Short-term effect of adding pelvic floor muscle training to bladder training for female uri- nary incontinence: a randomized controlled trial. Int Urogynecol J 2015;26:285-93.

21. Sherburn M, Bird M, Carey M, Bø K, Galea MP. Incontinence improves in older women after intensive pelvic floor muscle training: an assessor-blinded randomized controlled trial. Neurourol Urodyn 2011;30:317-24.

22. Hay-Smith EJ, Herderschee R, Dumoulin C, Herbison GP. Comparisons of approaches to pelvic floor muscle training for urinary incontinence in women. Cochrane Database Syst Rev 2011;(12): CD009508.

23. Alewijnse D, Mesters I, Metsemakers J, Adriaans J, van den Borne B. Predictors of intention to adhere to physiotherapy among women with urinary incontinence. Health Educ Res 2001;16:173-86. 